\title{
Complexity Growth Rate in Lovelock Gravity
}

\author{
Pablo A. Cano, ${ }^{1,2, *}$ Robie A. Hennigar, ${ }^{3, \dagger}$ and Hugo Marrochio, ${ }^{1,3,}$ \\ ${ }^{1}$ Perimeter Institute for Theoretical Physics, Waterloo, Ontario N2L 2Y5, Canada \\ ${ }^{2}$ Instituto de Física Teórica UAM/CSIC, C/ Nicolás Cabrera, 13-15, C.U. Cantoblanco, 28049 Madrid, Spain \\ ${ }^{3}$ Department of Physics and Astronomy, University of Waterloo, Waterloo, Ontario N2L 3G1, Canada
}

(Received 28 March 2018; revised manuscript received 26 June 2018; published 20 September 2018)

Using the complexity = action framework, we compute the late time growth of complexity for charged black holes in Lovelock gravity. Our calculation is facilitated by the fact that the null boundaries of the Wheeler-DeWitt patch do not contribute at late times and essential contributions coming from the joints are now understood. The late time growth rate reduces to a difference of internal energies associated with the inner and outer horizons, and in the limit where the mass is much larger than the charge, we reproduce the celebrated result of $2 M / \pi$ with corrections proportional to the highest Lovelock coupling in even (boundary) dimensions. We find in some cases a minimum mass below which complexity remains effectively constant, even if the black hole contains a nondegenerate horizon.

DOI: 10.1103/PhysRevLett.121.121602

The anti-de Sitter/conformal field theory (AdS/CFT) duality [1] has brought surprising insight to the nature of quantum gravity. The entanglement properties of the CFT suggest an emergence of the bulk geometry [2], with the RyuTakayanagi prescription for the entanglement entropy (EE) being the most explored example [3-5]. From the seminal works of Bekenstein $[6,7]$ and Hawking and coworkers [8,9], the thermodynamic properties of black hole geometries have raised intriguing questions about the possible microscopic structure of black holes. Understanding how these quantities are encoded in the dual CFT is presently an active area of study [10-22].

Recently, it has been observed that entanglement entropy may not be the appropriate observable to probe the interior degrees of freedom of black holes [23]. Based on intuition brought by tensor network models in holography [24,25], it was proposed that the complexity of the CFT state should encode information about the semiclassical geometry in the interior [26,27], motivated originally by the AMPS paradox [28]. Roughly speaking, complexity measures how hard it is to construct certain states in the theory from simple unentangled states and a few universal gates (see [29-37] for consideration on properties of complexity in the boundary theory). There are two related proposals conjectured to capture the complexity of the ground states of CFTs in holography: in this Letter, we focus on the "complexity = action" (CA) proposal $[38,39]$, as it permits

Published by the American Physical Society under the terms of the Creative Commons Attribution 4.0 International license. Further distribution of this work must maintain attribution to the author(s) and the published article's title, journal citation, and DOI. Funded by SCOAP . a conceptually straightforward generalization to include gravitational higher-curvature corrections (for exploration on "complexity = volume," see $[26,29,30,40,41])$. The CA proposal states that the complexity of the state is given by the Lorentzian action evaluated on the Wheeler-DeWitt (WDW) patch

$$
\mathcal{C}_{A}=\frac{I_{\mathrm{WDW}}}{\pi},
$$

which is the union of all spacelike hypersurfaces anchored at boundary times $t_{L}$ and $t_{R}$, as shown in Fig. 1.

Complexity is conjectured to continue increasing long after local thermal equilibrium is reached. The late time rate

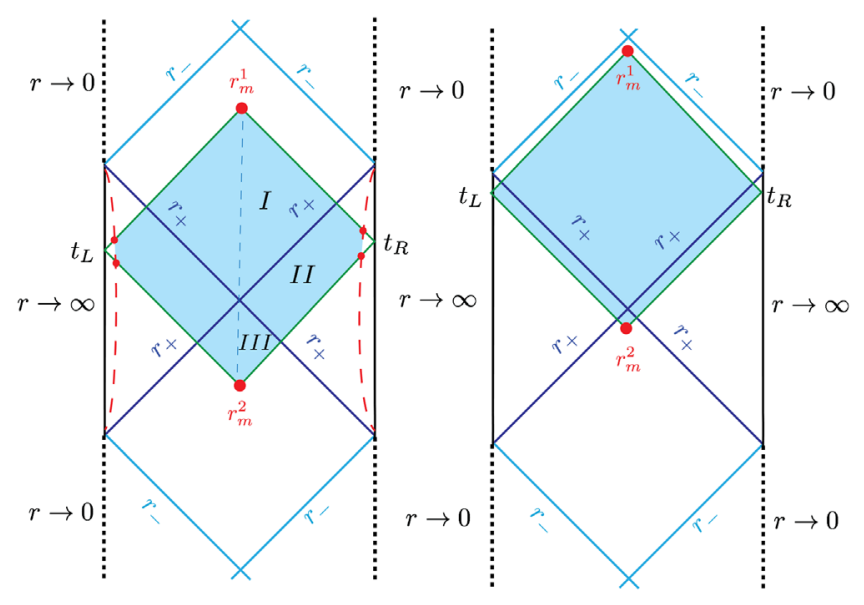

FIG. 1. The causal structure for a charged AdS black hole, with outer and inner horizons. The blue shaded region denotes the WDW patch, anchored at the boundary times $t_{L}=t_{R}=t / 2$. At late times, the null boundaries of the WDW patch approach the inner and outer horizons. 
of change is approximately $2 M / \pi$ in CA for a large class of uncharged black holes in Einstein gravity (EG) [38,39,42], suggesting a connection to Lloyd's bound on the rate of computation [43] and that black holes are the fastest scramblers in nature $[38,39,48]$. (Note, however, that it has been pointed out that the Lloyd bound can be violated in certain circumstances [40,44-47].)

While the properties of complexity in EG are now well understood in many situations, relatively little is known about its behavior in higher-derivative gravity $[49,50]$. In the context of AdS/CFT, higher-order curvature corrections in the bulk are dual to finite $N$ and finite coupling effects in the CFT [51-54]. The most relevant aspect of these corrections is that they allow us to explore more general holographic CFTs than those defined by Einstein gravity [55-64]. In this way, higher-order gravities allow us to identify universal relations valid for arbitrary theories, including, for example, general results about the EE [65-69]. Hence, one may wonder if, in the case of complexity, higher-curvature gravities could help us to see a possible hidden structure that is obscured when working with EG alone. Another interesting question is whether these theories could violate Lloyd's bound, analogously to how they violate the KovtunSon-Starinets bound on the shear viscosity to entropy density ratio $\eta / s \geq 1 /(4 \pi)[54,56,70-75]$, which was thought to be saturated in Einstein gravity holography [76].

One of the most suitable higher-curvature theories for holographic applications is Lovelock gravity $[55,56,62,71$, $75,77-79]$, due to unique properties such as second-order equations of motion [80,81] and the existence of a wellposed action functional $[82,83]$. Moreover, some of the Lovelock densities are actually predicted to appear in the effective low energy action of string theory [84], so they provide realistic corrections to the Einstein-Hilbert action.

In this Letter, we compute the complexity growth of black holes in Lovelock theory using the CA proposal. While CA presents no new conceptual challenges within higher-curvature gravity, it is nontrivial to identify the correct contributions to the action coming from the null boundaries and the joints in the WDW patch. It is important to identify these terms directly, since a limiting procedure based on timelike or spacelike surfaces is generically ambiguous [42]. We focus on charged black holes, since in this case the WDW patch approaches the inner and outer horizons at late time, allowing us to completely deduce the time-dependent structure of the null boundary terms. The contribution from the null joints was recently described in [85], and by taking them into account we identify an intriguing relation between the complexity growth at late times and the thermodynamic properties of the black hole.

Let us start by describing the theory and solutions of interest. The bulk action we consider is given by

$$
I_{\text {bulk }}=\int_{\mathcal{M}} d^{d+1} x \sqrt{|g|}\left(\mathcal{L}_{\text {grav }}-\frac{1}{4 g^{2}} F_{\mu \nu} F^{\mu \nu}\right),
$$

where $g$ is a constant, $F=d A$ is the Maxwell field strength, and $\mathcal{L}_{\text {grav }}$ is the Lovelock Lagrangian [80,81],

$$
\begin{aligned}
\mathcal{L}_{\text {grav }}= & \frac{1}{16 \pi G}\left[\frac{d(d-1)}{L^{2}}+R\right. \\
& \left.+\sum_{n=2}^{\lfloor d / 2\rfloor} \lambda_{n} \frac{(d-2 n) !}{(d-2) !}(-1)^{n} L^{2 n-2} \mathcal{X}_{2 n}\right],
\end{aligned}
$$

where the Euler densities $\mathcal{X}_{2 n}$ are given by

$$
\mathcal{X}_{2 n}=\frac{1}{2^{n}} \delta_{\nu_{1} \ldots \nu_{2 n}}^{\mu_{1} \ldots \mu_{2 n}} R_{\mu_{1} \mu_{2}}^{\nu_{1} \nu_{2}} \ldots R_{\mu_{2 n-1} \mu_{2 n}}^{\nu_{2 n-1} \nu_{2 n}}
$$

the generalized Kronecker symbol is defined as $\delta_{\nu_{1} \nu_{2} \ldots \nu_{r}}^{\mu_{1} \mu_{2} \ldots \mu_{r}}=r ! \delta_{\nu_{1}}^{\mu_{1}} \delta_{\nu_{2}}^{\mu_{2}} \ldots \delta_{\nu_{r}}^{\left.\mu_{r}\right]}$, and $\lambda_{n}$ are arbitrary dimensionless parameters.

Charged black holes in Lovelock gravity are known (see, for example, [86-88]) and, in general, the solution takes the form

$$
\begin{aligned}
d s^{2} & =-f(r) d t^{2}+\frac{d r^{2}}{f(r)}+r^{2} d \Sigma_{k, d-1}^{2}, \\
A & =d t \frac{g}{2 \sqrt{2 \pi G}} \sqrt{\frac{d-1}{d-2}} \frac{q}{r^{d-2}}=\phi(r) d t,
\end{aligned}
$$

where $d \Sigma_{k, d-1}^{2}$ characterizes the constant curvature transverse geometry, with $k=+1,0,-1$ denoting spherical, planar, and hyperbolic, respectively. The function $f$ satisfies the algebraic equation

$$
h\left(\frac{L^{2}(f(r)-k)}{r^{2}}\right)=\frac{\omega^{d-2} L^{2}}{r^{d}}-\frac{q^{2} L^{2}}{r^{2(d-1)}},
$$

with $h(x)$ given by the polynomial function

$$
h(x)=1-x+\sum_{n=2}^{\lfloor d / 2\rfloor} \lambda_{n} x^{n} .
$$

In these expressions, $q$ and $\omega$ are two integration constants that are related to the mass $M$ and the charge $Q$ $\left(Q=g^{-2} \int \star F\right)$ of the black hole according to

$$
\begin{aligned}
\omega^{d-2} & =\frac{16 \pi G M}{(d-1) \Omega_{k, d-1}}, \\
q & =\frac{g Q}{\Omega_{k, d-1}} \sqrt{\frac{8 \pi G}{(d-1)(d-2)}},
\end{aligned}
$$

where $\Omega_{k, d-1}$ is the (dimensionless) volume of the transverse space. Strictly speaking, $M$ and $Q$ are the mass and the charge only in the spherically symmetric case, $k=1$. 
In the noncompact cases, we should interpret $Q / \Omega_{k, d-1}$ as a charge density and $M / \Omega_{k, d-1}$ as a mass density.

To facilitate comparison with existing literature, we require that the causal structure of the charged black holes matches those in Einstein gravity, shown in Fig. 1. While this is always the case in Einstein gravity, in Lovelock gravity, the couplings must obey certain constraints in order to avoid a singularity before the inner horizon. For example, in Gauss-Bonnet gravity, a sufficient condition is to demand $0 \leq \lambda_{2}<1 / 4$, or $\lambda_{3}<-\left(\lambda_{2}\right)^{2} / 3$ in the cubic case. Within this constrained class of theories, we also note that, for $d$ even, there is a special behavior that happens when the energy is smaller than

$$
M_{\min }=\frac{(d-1) \Omega_{k, d-1}}{(16 \pi G)}(-k)^{d / 2} L^{d-2} \lambda_{d / 2} .
$$

Depending on the values of the couplings, neutral solutions with $M<M_{\min }$ are either naked singularities-thus, there are no black holes with mass below $M_{\text {min }}$ - or black holes with an inner horizon, as in the charged case (see, e.g., [89-91] for details).

We denote by $r_{+}$and $r_{-}$the event and inner horizon of the charged black hole where $f\left(r_{ \pm}\right)=0$. The temperature of the black hole is given in terms of the derivative of $f$ as $f^{\prime}\left(r_{+}\right)=4 \pi T_{+}$. On the other hand, the entropy is given by Wald's formula [92] or, equivalently, by the JacobsonMyers' result [93] [see Eq. (27)], and it reads

$S_{+}=\frac{r_{+}^{d-1} \Omega_{k, d-1}}{4 G}\left[1-\sum_{n=2}^{\lfloor d / 2\rfloor} \lambda_{n}\left(-\frac{k L^{2}}{r_{+}^{2}}\right)^{n-1} \frac{n(d-1)}{(d+1-2 n)}\right]$.

It will be useful to introduce as well the quantities $T_{-}$and $S_{-}$, defined in the analogous way at $r_{-}$.

These eternal black hole geometries should be dual to thermofield double (TFD) states, created by entangling each copy of the boundary CFT as [10]

$$
\begin{aligned}
\left|\operatorname{TFD}\left(t_{L}, t_{R}\right)\right\rangle= & Z^{-1 / 2} \sum_{\alpha, \sigma} e^{-i E_{\alpha}\left(t_{L}+t_{R}\right)} \\
& \times e^{-\left(E_{\alpha}-\mu Q_{\sigma}\right) /(2 T)}\left|E_{\alpha},-Q_{\sigma}\right\rangle\left|E_{\alpha}, Q_{\sigma}\right\rangle .
\end{aligned}
$$

We show in Fig. 1 a schematic Penrose diagram for charged black holes in asymptotic AdS spacetimes. In our conventions, both $t_{L}$ and $t_{R}$ increase towards the upper part of the diagram, so the boost symmetry in the state is encoded in $t_{R} \rightarrow t_{R}+\Delta t$ and $t_{L} \rightarrow t_{L}-\Delta t$. Therefore, we can focus on symmetric times $t_{L}=t_{R}=t / 2$ without loss of generality.

In Fig. 1, we recognize two coordinates that encode the time dependence, which we denote $r_{m}^{1}$ and $r_{m}^{2}$. We can identify how they depend on the boundary time $t$ by writing a transcendental equation involving the tortoise coordinate $r^{*}$, defined with $f(r)$ from eq. (5), such that

$$
r^{*}(r)=-\int_{r}^{\infty} \frac{d \tilde{r}}{f(\tilde{r})}, \quad \lim _{r \rightarrow \infty} r^{*}(r)=0 .
$$

The equations for $r_{m}^{1}$ and $r_{m}^{2}$ read

$$
\frac{t}{2}-r^{*}\left(r_{m}^{1}\right)=0, \quad \frac{t}{2}+r^{*}\left(r_{m}^{2}\right)=0 .
$$

As a consequence, the time derivative of these coordinates takes a simple form,

$$
\frac{d r_{m}^{1}}{d t}=\frac{f\left(r_{m}^{1}\right)}{2}, \quad \frac{d r_{m}^{2}}{d t}=-\frac{f\left(r_{m}^{2}\right)}{2} .
$$

Notice that, at late times, $r_{m}^{1}$ approaches the inner horizon $r_{-}$, while $r_{m}^{2}$ approaches the outer horizon $r_{+}$.

The action calculation on the WDW patch for black holes with the causal structure depicted in Fig. 1 has three possible contributions: a bulk integration, boundary contributions, and joint terms at $r_{m}^{1}$ and $r_{m}^{2}$,

$$
I=I_{\text {bulk }}+I_{\text {bdry }}+I_{\text {joint }} \text {. }
$$

Let us compute each of these terms.

First, we consider the contributions from the bulk action. If we express the integrand in the bulk action as a generic function of radius $r$, we have

$$
I_{\mathrm{bulk}}=\int_{\mathrm{WDW}} d^{d+1} x \sqrt{-g} \mathcal{L}=\int_{\mathrm{WDW}} d t d r I(r),
$$

where the function $I(r)$ will depend on the theory of gravity under consideration. For Lovelock gravity (2), it can be expressed as a total derivative $I(r)=d \mathcal{I}(r) / d r$, where

$$
\mathcal{I}(r)=\frac{\Omega_{k, d-1}}{16 \pi G}\left[-\frac{2(d-1) q^{2}}{r^{d-2}}+(d-1) \omega^{d-2}-r^{d-1} f^{\prime}(r)\left(1-\sum_{n=2}^{\lfloor d / 2\rfloor} \lambda_{n}\left(\frac{(f(r)-k) L^{2}}{r^{2}}\right)^{n-1} \frac{n(d-1)}{(d+1-2 n)}\right)\right] .
$$

The integration uses the fact that the Euler densities are total derivatives when evaluated on (5) [94], and the field equations (7) were used to simplify this result. 
Following [40], the bulk contribution to the action is the sum of three integrals,

$$
\begin{aligned}
& I_{\text {bulk }}^{\mathrm{I}}=2 \int_{r_{m}^{1}}^{r_{+}} I(r)\left(\frac{t}{2}-r^{*}(r)\right) d r, \\
& I_{\text {bulk }}^{\mathrm{II}}=-4 \int_{r_{+}}^{r_{\max }} I(r)\left(r^{*}(r)\right) d r, \\
& I_{\text {bulk }}^{\mathrm{III}}=2 \int_{r_{m}^{2}}^{r_{+}} I(r)\left(-\frac{t}{2}-r^{*}(r)\right) d r .
\end{aligned}
$$

Therefore, calculating the time derivative of the bulk term, using the properties of $r_{m}^{1}$ and $r_{m}^{2}$ in Eqs. (15) and (16), the answer depends on the differentiation with respect to time in the integrand, and the bulk contribution to the time derivative reads

$$
\frac{d I_{\mathrm{bulk}}}{d t}=\int_{r_{m}^{1}}^{r_{m}^{2}} I(r) d r=\left.\mathcal{I}(r)\right|_{r_{m}^{1}} ^{r_{m}^{2}}
$$

Then, in the late time limit $r_{m}^{2} \rightarrow r_{+}, r_{m}^{1} \rightarrow r_{-}$, and from (19), we observe that this contribution takes the appealing form

$$
\left.\frac{d I_{\mathrm{bulk}}}{d t}\right|_{t \rightarrow \infty}=\left.(M-T S-\phi Q)\right|_{r_{-}} ^{r_{+}},
$$

which is simply the difference of free energy one would associate with each horizon.

Let us now consider the null boundary terms and show that their time dependence vanishes. The null boundary terms for EG were described in $[42,95]$, but they are still unknown in Lovelock gravity. However, on general grounds, given a null segment $\mathcal{N}$ parametrized by $\lambda$ and with a transverse space metric $\sigma_{A B}$, the boundary contribution will have the form

$$
I_{\text {null }}=\int_{\mathcal{N}} d \lambda d x^{d-1} \sqrt{\sigma} \mathcal{Q}+\int_{\partial \mathcal{N}} d x^{d-1} \sqrt{\sigma} \mathcal{F}
$$

where, in analogy to the results in [85], we assume a possible contribution from the boundary of $\mathcal{N}$. This contribution could be equivalently understood as adding a total derivative to $\mathcal{Q}$. Here $\mathcal{Q}$ and $\mathcal{F}$ are some polynomial functions of intrinsic and extrinsic curvatures and of the parameter $\kappa$ defined as $k^{\alpha} \nabla_{\alpha} k_{\beta}=\kappa k_{\beta}$ (see [96] for the definition of the rest of the objects).

Now, let us ensure the time derivative of the null boundary term vanishes. Normalizing the null normal vectors on the WDW patch as, for instance, in [40], $r$ will be an affine parameter. Further, since the integrands will be functions of $r$ that we denote by $Q(r)$ (for the contribution along $\mathcal{N}$ ) and $G(r)$ (for the contribution along $\partial \mathcal{N}$ ), we will have

$$
\frac{d I_{\mathrm{null}}}{d t} \propto Q\left(r_{m}^{i}\right) \frac{d r_{m}^{i}}{d t}+\left.\frac{d G}{d r}\right|_{r=r_{m}^{i}} \frac{d r_{m}^{i}}{d t}
$$

Since these terms will be built from polynomials of intrinsic and extrinsic quantities, they will be finite (or vanishing) as $r_{m}^{i} \rightarrow r_{ \pm}$. Since the time derivatives of $r_{m}^{i}$ vanish in this limit, we are then assured that the null boundaries make no contributions to the time derivative at late times,

$$
\left.\frac{d I_{\text {null }}}{d t}\right|_{t \rightarrow \infty}=0 .
$$

Finally, we consider the contribution to the rate of change of joints at $r_{m}^{1}$ and $r_{m}^{2}$. The joint terms for intersections of null boundaries were described in [85], where it was found that they are given by

$$
I_{\text {joint }}=\frac{1}{2 \pi} \int_{\mathcal{C}} d \sigma a \rho_{\mathrm{JM}},
$$

where the parameter $a$ is the same that appears in Einstein gravity [42], and $\rho_{\mathrm{JM}}$ is the Jacobson-Myers entropy [93] associated with the codimension 2 surface $\mathcal{C}$,

$\rho_{\mathrm{JM}}=\frac{1}{4 G}\left[1+\sum_{n=2}^{\lfloor d / 2\rfloor} n \lambda_{n} \frac{(d-2 n) !}{(d-2) !}(-1)^{n} L^{2 n-2} \hat{\mathcal{X}}_{2(n-1)}\right]$,

where $\hat{\mathcal{X}}_{2(n-1)}$ is the $(n-1)$ th Euler density of the induced metric. For the case depicted in Fig. 1, the joint contributions take the form

$$
I_{\text {joint }}=\frac{1}{2 \pi}\left[S\left(r_{m}^{1}\right) a\left(r_{m}^{1}\right)+S\left(r_{m}^{2}\right) a\left(r_{m}^{2}\right)\right],
$$

where $S(r)$ evaluates the entropy at the horizons. Following the conventions of, for instance, [40,97], the function $a$ at joints like those of $r_{m}^{1}$ and $r_{m}^{2}$ is given by

$$
a(r)=-\log \left(\frac{|f(r)|}{\alpha^{2}}\right),
$$

where $\alpha$ is an arbitrary constant in the normalization of the null vector with respect to a boundary timelike vector, as described in [42].

The time derivative of the joint contributions to the action takes a compact and simple form at late times. For instance, at $r_{m}^{1}$ the time derivative of $S a$ takes the general form

$\left.\frac{d(S a)}{d t}\right|_{r=r_{m}^{1}}=\left.\frac{1}{2} a\left(r_{m}^{1}\right) f\left(r_{m}^{1}\right) \frac{d S(r)}{d r}\right|_{r=r_{m}^{1}}-\frac{1}{2} S\left(r_{m}^{1}\right) f^{\prime}\left(r_{m}^{1}\right)$,

where we used (16) and (29). The first contribution to the right-hand side of Eq. (30) vanishes when $r_{m}^{1}$ approaches 
$r_{-}$, since the derivative of $S(r)$ is finite and $\lim _{r_{m}^{1} \rightarrow r_{-}} a\left(r_{m}^{1}\right)$ $f\left(r_{m}^{1}\right)=0$. On the other hand, $f^{\prime}\left(r_{m}^{1}\right) /(4 \pi)$ approaches the "temperature" $T_{-}$of the horizon when evaluated at $r_{-}$. An analogous computation holds for $r_{m}^{2}$ approaching $r_{+}$. Then, the time derivative of the joint contribution takes the simple form

$$
\left.\frac{d I_{\text {joint }}}{d t}\right|_{t \rightarrow \infty}=\left.T S\right|_{r_{-}} ^{r_{+}}
$$

Putting together the results (22) and (31), we have the late time complexity growth rate,

$$
\left.\pi \frac{d \mathcal{C}_{A}}{d t}\right|_{t \rightarrow \infty}:=\pi \dot{\mathcal{C}}_{A}=\phi_{-} Q-\phi_{+} Q
$$

which holds for Lovelock theory of any order and in any dimension.

Our result can be expressed in another useful form. Introducing the dimensionless parameters $y:=r_{-} / r_{+}$and $z:=L / r_{+}$, we can write

$$
\dot{\mathcal{C}}_{A}=\frac{2 M}{\pi}\left[\frac{\left(h_{+}-y^{d} h_{-}\right)\left(1-y^{d-2}\right)}{h_{+}-y^{2(d-1)} h_{-}}\right],
$$

with $h_{+}:=h\left(-k z^{2}\right)$ and $h_{-}:=h\left(-k z^{2} / y^{2}\right)$. We can then consider two limits of interest. First, we see that in the extremal limit $y \rightarrow 1$ and we get $\dot{\mathcal{C}}_{A} \rightarrow 0$. Second, we can consider the limit of vanishing charge, $y \rightarrow 0$. Here we must take note of the following result,

$$
\lim _{y \rightarrow 0} y^{d} h_{-}= \begin{cases}0 & \text { for } d \text { odd } \\ (-k)^{d / 2} \lambda_{d / 2} z^{d} & \text { for } d \text { even }\end{cases}
$$

and so we obtain in the uncharged limit

$$
\dot{\mathcal{C}}_{A}=\frac{2\left(M-M_{\min }\right)}{\pi},
$$

where it is understood that the correction to $2 M$ is only present for even $d$ and $k$ nonzero [98] [see (11) for the expression of $\left.M_{\text {min }}\right]$. Strictly speaking, we should consider this result to hold in a regime where the mass is much larger than the charge, but the charge is still large enough that the inner horizon is not "close" to the singularity.

The vanishing of $\dot{\mathcal{C}}_{A}$ at extremality is in line with results from Einstein gravity [38,39,42], while the appearance of $M_{\min }$ at small charge leads to new features. In cases where $M_{\text {min }}=0$, the growth rate comes closest to saturating Lloyd's bound as $y \rightarrow 0$, as in Einstein gravity. However, for $M_{\min } \neq 0$, the growth rate may reach a maximum (less than $2 M / \pi$ ) for nonzero $y$.

There are two possible scenarios in the neutral limit. The first possibility is that when $M=M_{\min }$ the black hole becomes zero size and then the correction in (35) ensures that $\dot{\mathcal{C}}_{A}=0$ in that case. For $M<M_{\min }$ there is no black hole. The second possibility is that when $M<M_{\min }$ the black hole develops an internal horizon, and in that case $\dot{\mathcal{C}}_{A}=0$ even if the black hole has nonvanishing temperature. Note that this same result can be obtained directly from the uncharged solution, due to the two horizon causal structure, and so is true irrespective of the uncharged limit presented above. Therefore, there is a minimum mass below which black holes do not increase complexity. Since it is usually claimed that black holes are the fastest computers on nature, this result would suggest that there is a minimum mass required to perform computation.

To summarize, we have carried out the first general calculation within the complexity $=$ action framework, taking higher-curvature corrections into account. In the late time limit, we argued that, due to the WDW patch approaching the inner and outer horizons of charged black holes, the null boundary terms are unimportant and the calculation requires only the bulk and joint terms, which are now understood [85]. For spherical black holes in GaussBonnet gravity, our results agree with those in [49], though we note that there they were computed using other methods, taking the limit of spacelike and timelike boundaries.

The complexity growth rate reduced beautifully to thermodynamic expressions,

$\pi \dot{\mathcal{C}}_{A}=\left(F_{+}+T_{+} S_{+}\right)-\left(F_{-}+T_{-} S_{-}\right)=U_{+}-U_{-}$,

with $F_{ \pm}$as the free energy associated with each horizon and $U_{ \pm}$as the internal energy [101]. This result is at once surprising and suggestive. It shows that the results first obtained in $[38,39]$ are of incredibly broad scope, holding their form even in the presence of higher-curvature (finite $N$ ) corrections. Our calculation shows in a very transparent way the origin of this result: the bulk contribution is always the free energy and the joint contribution is always $T S$. From this, one might expect that this expression is of broader applicability than the situation considered here, and may in fact hold for any two horizon configuration. At the very least, this suggests a deep connection between the late time growth of complexity and black hole thermodynamics that merits further exploration, as for instance in [31].

In principle, our methods will also work directly in the uncharged case. However, as noted in $[39,49]$, there are subtleties related to the way in which one regularizes the hypersurface above the singularity-we will discuss this further in forthcoming work [105]. In the charged case, these problems are, in general, not present since the singularity is hidden behind the inner horizon.

Lastly, let us note that, while we have taken an important first step toward understanding the role of higher-curvature theories in the framework of holographic complexity, there remains much to explore. For example, we expect to see corrections to the complexity of formation [106]. Furthermore, it would be interesting to explore the corrections to the full time dependence of complexity. In [40] it 
was found that the late time rate $2 M / \pi$ is approached from above, rather than from below as Lloyd's bound would suggest. Addressing these questions would require full knowledge of the null boundary terms, which are still unknown for Lovelock gravity.

We are pleased to thank Rob Myers and Robb Mann for useful conversations and comments. The work of P. A. C. is funded by Fundación la Caixa through a "la Caixa-Severo Ochoa" international predoctoral grant. The work of P. A. C. was also supported by the MINECO/FEDER, UE Grant No. FPA2015-66793-P and by the Spanish Research Agency (Agencia Estatal de Investigación) through the grant IFT Centro de Excelencia Severo Ochoa SEV-2016-0597. P. A.C. also thanks the Perimeter Institute "Visiting Graduate Fellows" program. Research at Perimeter Institute is supported by the Government of Canada through the Department of Innovation, Science and Economic Development and by the Province of Ontario through the Ministry of Research, Innovation and Science.

*pablo.cano@uam.es

†hennigar@uwaterloo.ca

"hmarrochio@perimeterinstitute.ca

[1] J. M. Maldacena, Int. J. Theor. Phys. 38, 1113 (1999); Adv. Theor. Math. Phys. 2, 231 (1998).

[2] M. Van Raamsdonk, Gen. Relativ. Gravit. 42, 2323 (2010); Int. J. Mod. Phys. D 19, 2429 (2010).

[3] S. Ryu and T. Takayanagi, J. High Energy Phys. 08 (2006) 045.

[4] T. Nishioka, S. Ryu, and T. Takayanagi, J. Phys. A 42, 504008 (2009).

[5] V. E. Hubeny, M. Rangamani, and T. Takayanagi, J. High Energy Phys. 07 (2007) 062.

[6] J. D. Bekenstein, Phys. Rev. D 7, 2333 (1973).

[7] J. D. Bekenstein, Phys. Rev. D 9, 3292 (1974).

[8] J. M. Bardeen, B. Carter, and S. W. Hawking, Commun. Math. Phys. 31, 161 (1973).

[9] S. W. Hawking, Commun. Math. Phys. 43, 167 (1975).

[10] J. M. Maldacena, J. High Energy Phys. 04 (2003) 021.

[11] H. Casini, M. Huerta, and R. C. Myers, J. High Energy Phys. 05 (2011) 036.

[12] N. Lashkari, M. B. McDermott, and M. Van Raamsdonk, J. High Energy Phys. 04 (2014) 195.

[13] T. Hartman and J. Maldacena, J. High Energy Phys. 05 (2013) 014.

[14] T. Faulkner, A. Lewkowycz, and J. Maldacena, J. High Energy Phys. 11 (2013) 074.

[15] T. Faulkner, M. Guica, T. Hartman, R. C. Myers, and M. Van Raamsdonk, J. High Energy Phys. 03 (2014) 051.

[16] J. Maldacena and L. Susskind, Fortschr. Phys. 61, 781 (2013).

[17] B. Swingle and M. Van Raamsdonk, arXiv:1405.2933.

[18] A. Almheiri, X. Dong, and D. Harlow, J. High Energy Phys. 04 (2015) 163.

[19] T. Jacobson, Phys. Rev. Lett. 116, 201101 (2016).
[20] F. Pastawski, B. Yoshida, D. Harlow, and J. Preskill, J. High Energy Phys. 06 (2015) 149.

[21] B. Czech, L. Lamprou, S. McCandlish, and J. Sully, J. High Energy Phys. 10 (2015) 175.

[22] J. de Boer, M. P. Heller, R. C. Myers, and Y. Neiman, Phys. Rev. Lett. 116, 061602 (2016).

[23] L. Susskind, Fortschr. Phys. 64, 49 (2016).

[24] G. Vidal, Phys. Rev. Lett. 101, 110501 (2008).

[25] B. Swingle, Phys. Rev. D 86, 065007 (2012).

[26] L. Susskind, Fortschr. Phys. 64, 24 (2016); 64, 24(E) (2016).

[27] D. Harlow and P. Hayden, J. High Energy Phys. 06 (2013) 085.

[28] A. Almheiri, D. Marolf, J. Polchinski, and J. Sully, J. High Energy Phys. 02 (2013) 062.

[29] D. Stanford and L. Susskind, Phys. Rev. D 90, 126007 (2014).

[30] L. Susskind and Y. Zhao, arXiv:1408.2823.

[31] A. R. Brown and L. Susskind, Phys. Rev. D 97, 086015 (2018).

[32] R. Jefferson and R. C. Myers, J. High Energy Phys. 10 (2017) 107.

[33] S. Chapman, M. P. Heller, H. Marrochio, and F. Pastawski, Phys. Rev. Lett. 120, 121602 (2018).

[34] P. Caputa, N. Kundu, M. Miyaji, T. Takayanagi, and K. Watanabe, Phys. Rev. Lett. 119, 071602 (2017).

[35] P. Caputa, N. Kundu, M. Miyaji, T. Takayanagi, and K. Watanabe, J. High Energy Phys. 11 (2017) 097.

[36] R.-Q. Yang, Phys. Rev. D 97, 066004 (2018).

[37] R. Khan, C. Krishnan, and S. Sharma, arXiv:1801.07620.

[38] A. R. Brown, D. A. Roberts, L. Susskind, B. Swingle, and Y. Zhao, Phys. Rev. Lett. 116, 191301 (2016).

[39] A. R. Brown, D. A. Roberts, L. Susskind, B. Swingle, and Y. Zhao, Phys. Rev. D 93, 086006 (2016).

[40] D. Carmi, S. Chapman, H. Marrochio, R. C. Myers, and S. Sugishita, J. High Energy Phys. 11 (2017) 188.

[41] J. Couch, W. Fischler, and P. H. Nguyen, J. High Energy Phys. 03 (2017) 119.

[42] L. Lehner, R. C. Myers, E. Poisson, and R. D. Sorkin, Phys. Rev. D 94, 084046 (2016).

[43] S. Lloyd, Nature (London) 406, 1047 (2000).

[44] J. Couch, S. Eccles, W. Fischler, and M.-L. Xiao, J. High Energy Phys. 03 (2018) 108.

[45] B. Swingle and Y. Wang, arXiv:1712.09826.

[46] M. Alishahiha, A. F. Astaneh, M. R. M. Mozaffar, and A. Mollabashi, J. High Energy Phys. 07 (2018) 042.

[47] Y.-S. An and R.-H. Peng, Phys. Rev. D 97, 066022 (2018).

[48] Y. Sekino and L. Susskind, J. High Energy Phys. 10 (2008) 065 .

[49] R.-G. Cai, S.-M. Ruan, S.-J. Wang, R.-Q. Yang, and R.-H. Peng, J. High Energy Phys. 09 (2016) 161.

[50] M. Alishahiha, A. Faraji Astaneh, A. Naseh, and M. H. Vahidinia, J. High Energy Phys. 05 (2017) 009.

[51] M. T. Grisaru, A. E. M. van de Ven, and D. Zanon, Phys. Lett. B 173, 423 (1986).

[52] D. J. Gross and E. Witten, Nucl. Phys. B277, 1 (1986).

[53] S. S. Gubser, I. R. Klebanov, and A. A. Tseytlin, Nucl. Phys. B534, 202 (1998).

[54] A. Buchel, J. T. Liu, and A. O. Starinets, Nucl. Phys. B707, 56 (2005). 
[55] X. O. Camanho and J. D. Edelstein, J. High Energy Phys. 04 (2010) 007.

[56] J. de Boer, M. Kulaxizi, and A. Parnachev, J. High Energy Phys. 03 (2010) 087.

[57] A. Buchel, R. C. Myers, and A. Sinha, J. High Energy Phys. 03 (2009) 084.

[58] D. M. Hofman and J. Maldacena, J. High Energy Phys. 05 (2008) 012.

[59] D. M. Hofman, Nucl. Phys. B823, 174 (2009).

[60] S. Nojiri and S. D. Odintsov, Int. J. Mod. Phys. A 15, 413 (2000).

[61] M. Blau, K. S. Narain, and E. Gava, J. High Energy Phys. 09 (1999) 018.

[62] A. Buchel, J. Escobedo, R. C. Myers, M. F. Paulos, A. Sinha, and M. Smolkin, J. High Energy Phys. 03 (2010) 111.

[63] R. C. Myers, M. F. Paulos, and A. Sinha, J. High Energy Phys. 08 (2010) 035.

[64] P. Bueno, P. A. Cano, and A. Ruiperez, J. High Energy Phys. 03 (2018) 150.

[65] R. C. Myers and A. Sinha, J. High Energy Phys. 01 (2011) 125.

[66] R. C. Myers and A. Sinha, Phys. Rev. D 82, 046006 (2010).

[67] M. Mezei, Phys. Rev. D 91, 045038 (2015).

[68] P. Bueno, R. C. Myers, and W. Witczak-Krempa, Phys. Rev. Lett. 115, 021602 (2015).

[69] P. Bueno and R. C. Myers, J. High Energy Phys. 08 (2015) 068.

[70] Y. Kats and P. Petrov, J. High Energy Phys. 01 (2009) 044.

[71] M. Brigante, H. Liu, R. C. Myers, S. Shenker, and S. Yaida, Phys. Rev. D 77, 126006 (2008).

[72] R. C. Myers, M. F. Paulos, and A. Sinha, Phys. Rev. D 79, 041901 (2009).

[73] R.-G. Cai, Z.-Y. Nie, and Y.-W. Sun, Phys. Rev. D 78, 126007 (2008).

[74] X.-H. Ge, Y. Matsuo, F.-W. Shu, S.-J. Sin, and T. Tsukioka, J. High Energy Phys. 10 (2008) 009.

[75] X. O. Camanho, J. D. Edelstein, and M. F. Paulos, J. High Energy Phys. 05 (2011) 127.

[76] P. K. Kovtun, D. T. Son, and A. O. Starinets, Phys. Rev. Lett. 94, 111601 (2005).

[77] J. de Boer, M. Kulaxizi, and A. Parnachev, J. High Energy Phys. 06 (2010) 008.

[78] X. O. Camanho and J. D. Edelstein, J. High Energy Phys. 06 (2010) 099.

[79] J. de Boer, M. Kulaxizi, and A. Parnachev, J. High Energy Phys. 07 (2011) 109.

[80] D. Lovelock, Aequationes mathematicae 4, 127 (1970).

[81] D. Lovelock, J. Math. Phys. 12, 498 (1971).

[82] R. C. Myers, Phys. Rev. D 36, 392 (1987).
[83] C. Teitelboim and J. Zanelli, Classical Quantum Gravity 4, L125 (1987).

[84] R. Metsaev and A. Tseytlin, Nucl. Phys. B293, 385 (1987).

[85] P. A. Cano, Phys. Rev. D 97, 104048 (2018).

[86] J. T. Wheeler, Nucl. Phys. B273, 732 (1986).

[87] M. Cvetic, S. Nojiri, and S. D. Odintsov, Nucl. Phys. B628, 295 (2002).

[88] A. M. Frassino, D. Kubiznak, R. B. Mann, and F. Simovic, J. High Energy Phys. 09 (2014) 080.

[89] R. C. Myers and J.Z. Simon, Phys. Rev. D 38, 2434 (1988).

[90] R.-G. Cai, Phys. Lett. B 582, 237 (2004).

[91] N. Deppe, A. Kolly, A. Frey, and G. Kunstatter, Phys. Rev. Lett. 114, 071102 (2015).

[92] R. M. Wald, Phys. Rev. D 48, R3427 (1993).

[93] T. Jacobson and R. C. Myers, Phys. Rev. Lett. 70, 3684 (1993).

[94] S. Deser and A. V. Ryzhov, Classical Quantum Gravity 22, 3315 (2005).

[95] K. Parattu, S. Chakraborty, B. R. Majhi, and T. Padmanabhan, Gen. Relativ. Gravit. 48, 94 (2016).

[96] I. Vega, E. Poisson, and R. Massey, Classical Quantum Gravity 28, 175006 (2011).

[97] D. Carmi, R. C. Myers, and P. Rath, J. High Energy Phys. 03 (2017) 118.

[98] It would be interesting to investigate further the connection between topological effects and complexity as explored in [99]. One possible direction would be in the context of Lovelock-Chern-Simons theories [100].

[99] Z. Fu, A. Maloney, D. Marolf, H. Maxfield, and Z. Wang, J. High Energy Phys. 02 (2018) 072.

[100] J. Crisostomo, R. Troncoso, and J. Zanelli, Phys. Rev. D 62, 084013 (2000).

[101] Let us note that this relation was also noticed in [102], where it was argued to hold, in general. However, the argument relied on a number of assumptions whose validity is difficult to assess. This work was done in the framework of black hole chemistry $[103,104]$, where the mass is interpreted as the enthalpy of spacetime (see also [41]).

[102] H. Huang, X.-H. Feng, and H. Lu, Phys. Lett. B 769, 357 (2017).

[103] D. Kastor, S. Ray, and J. Traschen, Classical Quantum Gravity 26, 195011 (2009).

[104] D. Kubiznak, R. B. Mann, and M. Teo, Classical Quantum Gravity 34, 063001 (2017).

[105] P. A. Cano, R. A. Hennigar, and H. Marrochio (to be published).

[106] S. Chapman, H. Marrochio, and R. C. Myers, J. High Energy Phys. 01 (2017) 062. 